\title{
Trans-sialidase Protein as a Potential Serological Marker for African Trypanosomiasis
}

\author{
Ana Filipa Teixeira ${ }^{1}$ João Gomes Pereira ${ }^{1} \cdot$ Sónia Pestana-Ascensão ${ }^{1} \cdot$ Marcelo Sousa Silva ${ }^{1,2,3,4}$ [0
}

Published online: 2 January 2019

(c) Springer Science+Business Media, LLC, part of Springer Nature 2019

\begin{abstract}
Trypanosoma brucei is the etiological agent of African trypanosomiasis responsible for human and animal infections. T. bru$c e i$ is transmitted by infected tsetse flies. There is no vaccine for the disease and drugs available for treatment are inefficient and high toxicity. In this context, it is a priority to find antigenic targets suitable for the development of new diagnostic tools, drugs and vaccines. In this work, we report that mice infected with $T$. b. brucei produce antibodies against trans-sialidase recombinant protein (TS). In addition, we also demonstrate that bloodstream $T$. $b$. brucei express messenger RNA related to the TS gene. Collectively, our data strongly suggest that bloodstream forms of $T$. $b$. brucei also express the TS gene, that to date was described only in the procyclic forms of the T. $b$. brucei. In conclusion, these results highlight the importance of TS protein as a possible antigen target during infection caused by $T$. b. brucei.
\end{abstract}

Keywords Trans-sialidase $\cdot$ Trypanosoma brucei $\cdot$ African trypanosomiasis $\cdot$ Antigenic targets $\cdot$ Serological markers

\section{Introduction}

Trypanosoma brucei is the etiological agent of African trypanosomiasis (AT) or sleeping sickness in humans and Nagana in animals. AT is a neglected disease caused by $T$. brucei, a protozoan parasite transmitted by infected vector tsetse flies. Two main evolutionary forms characterize the life cycle of T. brucei; the procyclic forms present in the vector, and the bloodstream forms present in mammalian

Ana Filipa Teixeira and João Gomes Pereira have contributed equally to this work.

Marcelo Sousa Silva

mssilva@ihmt.unl.pt

1 Global Health and Tropical Medicine, Institute of Hygiene and Tropical Medicine, Universidade Nova de Lisboa, Lisbon, Portugal

2 Immunoparasitology Laboratory, Department of Clinical and Toxicological Analysis, Federal University of Rio Grande do Norte, Natal, Brazil

3 Programa de Pós-graduação em Bioquímica, Centro de Biociências, Federal University of Rio Grande do Norte, Natal, Brazil

4 Programa de Pós-graduação em Ciências Farmacêuticas, Centro de Ciências da Saúde, Federal University of Rio Grande do Norte, Natal, Brazil hosts. In the mammalian hosts parasites assume different morphologies and expression patterns [1].

In T. brucei bloodstream forms, the parasite's membrane is covered mainly by variant surface glycoproteins (VSG), a family of variable proteins that allows trypanosomes to sur

vive during infection in the mammalian host, causing immune system disorders and symptoms of chronic diseases [2]. In the context of procyclic forms, parasites are coated with procyclin, a membrane protein that replaces VSG from the bloodstream forms of T. brucei. VSG coat is constantly changing by antigenic variation, which is a mechanism of immune evasion where VSG molecules are constantly being produced and released in different serotypes [3].

Besides the VSG molecules in bloodstream stages and the procyclin in procyclic forms, T. brucei also present other membrane components that interact with the host during infection. One of these components is the trans-sialidase protein (TS); an enzyme well described in the procyclic forms of T. brucei [4-7]. However, the presence of TS protein is not completely resolved in bloodstream forms of $T$. brucei. Conversely, TS protein has been very well described in T. cruzi protozoan, the etiological agent of Chagas disease also denominated of American trypanosomiasis [8-10]. In $T$. cruzi, the TS component is an important toxin, serological maker, and virulence factor during infection, representing a promising target for vaccine development [11-14]. 
The TS protein is a membrane-associated enzyme used by trypanosomes to obtain sialic acid from their host, and to incorporate it into their cell-surface glycoproteins [8]. Thus, TS is responsible for transferring sialic acid molecules from the host's cell surface to the acceptor molecules on the parasites' surface, a phenomenon known as sialylation, which occurs in the different stages of development of the parasite, contributing to virulence of the T. cruzi [15].

The first isolation and characterization of TS protein was reported in $T$. cruzi [8], followed by an equivalent enzyme from cultured procyclic forms of the African trypanosomes species $T$. brucei and T. congolense [6, 16-18]. In contrast to T. cruzi parasite, TS has not yet been resolved in bloodstream forms of $T$. brucei, being reported to express in the procyclic forms only. Recently, Ammar et al. [19] have detected TS activity in bloodstream forms of T. b. gambiense.

In our previous work, we suggested for the first time the presence of TS protein also in bloodstream T. $b$. bruce $i$ [20]. We demonstrated that mice, immunized by DNA vaccine encoding TS gene, produce anti-TS antibodies able to recognize a total crude extract of bloodstream forms of $T$. b. brucei. Interestingly, this immunization process is able to elicit partial protection of mice challenged with infective T. b. brucei parasites.

In the present work, we report for the first time that Balb-C mice infected with T. b. brucei produced anti-TS antibodies that recognized TS recombinant protein. In addition, T. $b$. brucei bloodstream stages, purified from infected mice, expressed mRNA for TS gene expression. These results support the importance of TS as a possible antigenic serological marker during infection caused by $T$. b. brucei parasites.

\section{Results}

To date, the literature has reported some inconsistence regarding the presence of TS protein only in the procyclic forms of $T$. b. brucei. In order to verify the presence of this enzyme also in the bloodstreams $T$. b. brucei, bloodstream parasites were purified from blood of infected mice with T. b. brucei GVR 35/1.5. Figure 1 shows the purification process of the parasites obtained from blood of infected mice. Whole blood from infected mice was fixed on slides and subsequently stained by Giemsa (Fig. 1a). Then, blood samples were submitted to DEAE-cellulose chromatography and the bloodstreams forms of $T$. b. brucei were obtained, as shown in Fig. 1b. We visualized by light microscopy that the blood cells were eliminated during the chromatographic process, and that the bloodstream forms of $T . b$. bruce $i$ were efficiently eluted and purified from the whole blood from infected mice (Fig. 1). Purified parasites were then washed in PBS-glucose and subsequently frozen at $-80{ }^{\circ} \mathrm{C}$ until the time of use. This preparation, containing only bloodstream $T$. $b$. brucei, was used to obtain genomic DNA, total RNA and crude protein extract of bloodstream forms of $T . b$. brucei.

In order to verify TS gene expression in the bloodstream forms of T. b. brucei, parasites purified from blood of infected mice were submitted to extraction and purification of genomic DNA, and total RNA by commercial kit (Bioline-UK). cDNA of bloodstream forms of $T . b$. brucei, obtained by reverse transcriptase-PCR, was then used as a template for PCR amplification of the complete TS gene sequence $(2,316 \mathrm{bp})$ and the first $1.416 \mathrm{bp}$ fragment of the TS gene, here named nTS. Figure 2 shows the illustrative representation of the TS gene sequence, the
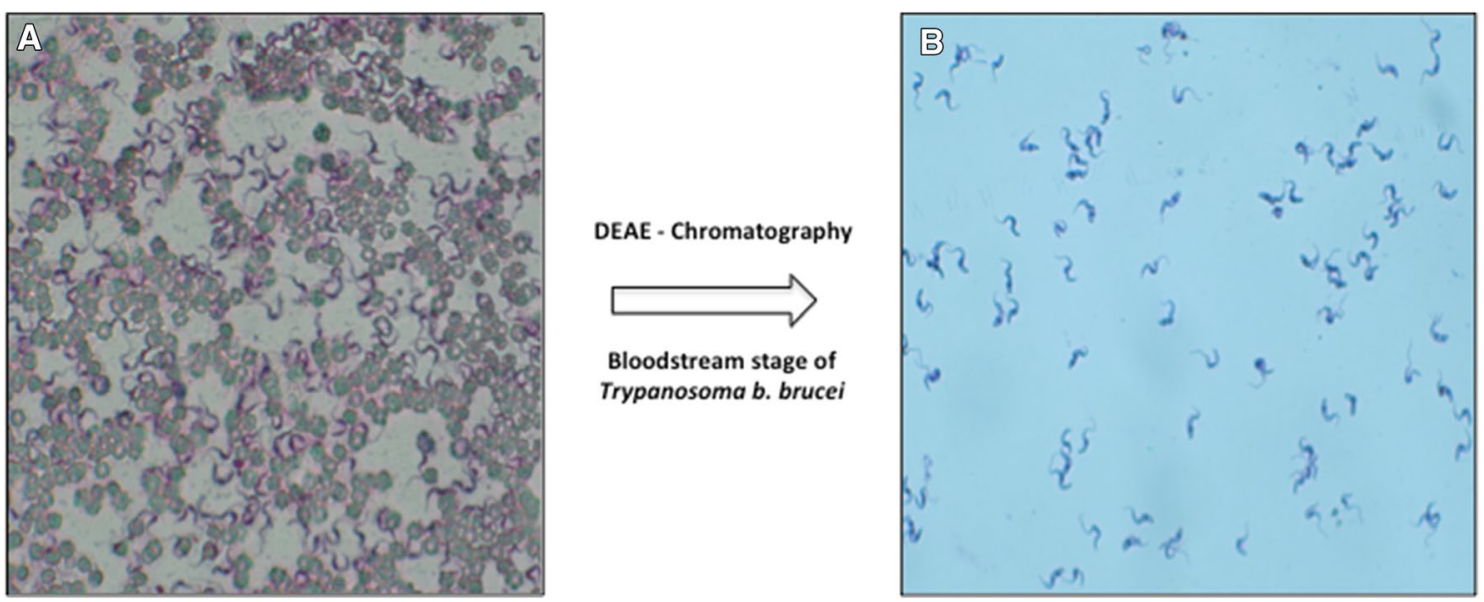

Fig. 1 Purification process by DEAE-Sepharose chromatography of bloodstream forms of $T$. $b$. brucei obtained from infected Balb-C mice. a Whole blood from infected mice; $\mathbf{b}$ bloodstreams forms of the parasites obtained after chromatography, both slides were fixed and stained by Giemsa's staining (optical microscopy 400x) 


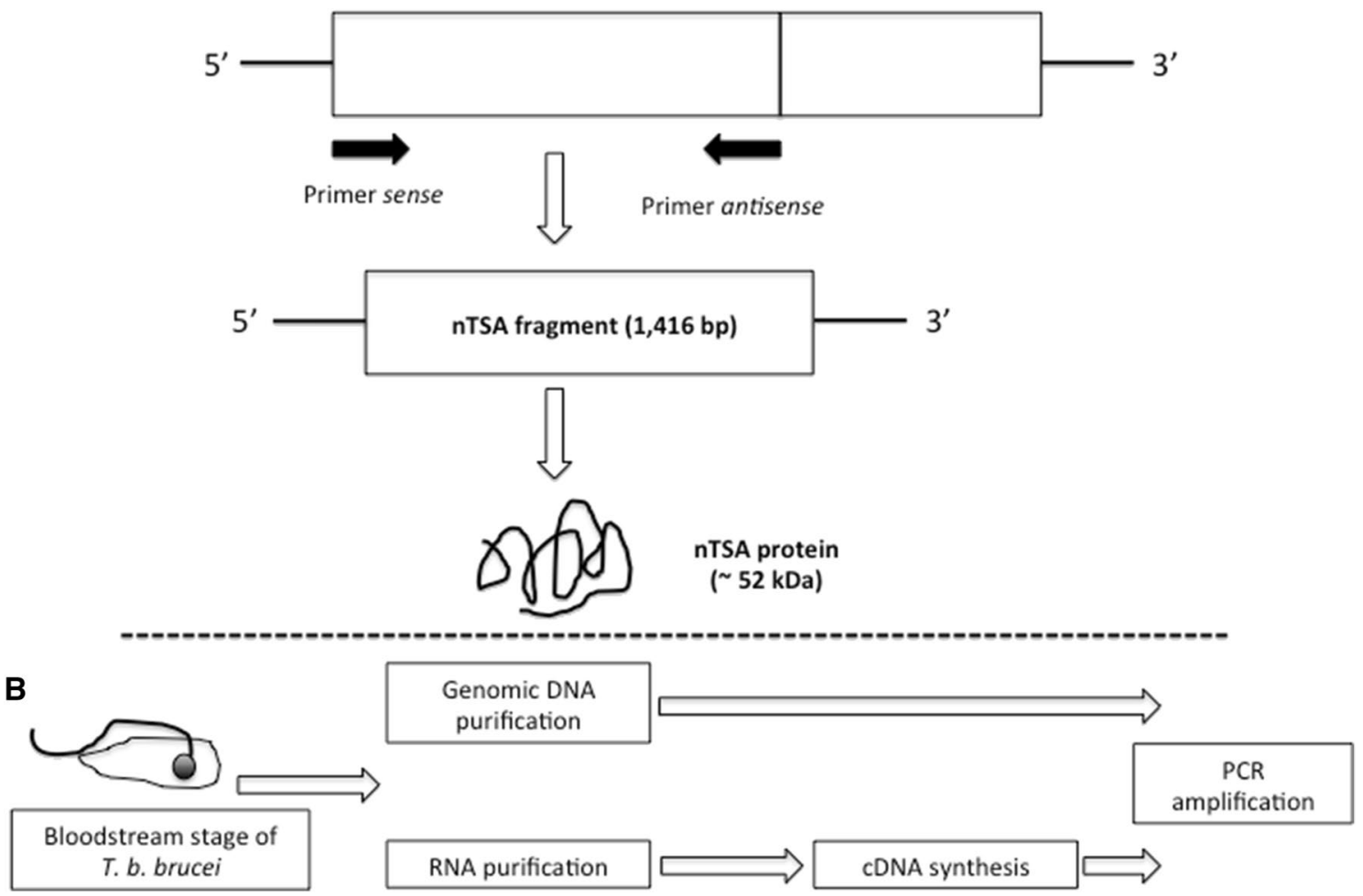

Fig. 2 Representative illustration of the cloning strategy of TS gene and purification of genomic DNA and mRNA of bloodstream forms of $T$. $b$. brucei parasites. a Cloning strategy of trans-sialidase gene

TS fragment (Fig. 2a) and the experimental strategy used to demonstrate the presence of TS gene expression in the bloodstream forms of T. b. brucei (Fig. 2b).

Afterwards, in order to verify the presence of specific mRNA for TS gene of bloodstream forms of $T$. $b$. bruce $i$, specific primers were used for amplification of TS gene by conventional and non-quantitative PCR. Simultaneously, the 1,416 bp fragment of TS gene was also amplified. In both cases, cDNA obtained from bloodstream forms of $T$. $b$. bruce $i$ was used as a template for amplification reactions. As shown in Fig. 3, bloodstream forms of T. b. bruce $i$ have specific mRNA for the TS gene (2,316 bp-lane 3), which is also amplified by PCR for the nTS fragment $(1,426 \mathrm{bp}-$ lane 2). As a positive control of PCR, we used specific primers to amplify a stage-specific gene of bloodstream forms of $T$. $b$. brucei, an Invariant Surface Glycoprotein gene (ISG), about $1,572 \mathrm{bp}$ (lane 1). Figure 3 also shows that ISG and TS genes are not amplified by conventional PCR, when the template consists only of RNA obtained of bloodstream forms of $T$. b. brucei (lanes 4-6). Likewise, both genes are amplified by conventional PCR when the template is genomic DNA obtained from bloodstream forms of T. b. brucei (lanes 7-8).

After confirming the presence of the TS gene in the bloodstream forms of $T$. $b$. brucei by molecular techniques and nTS sequences. b Purification of the genomic DNA and mRNA from bloodstream forms of T. b. brucei

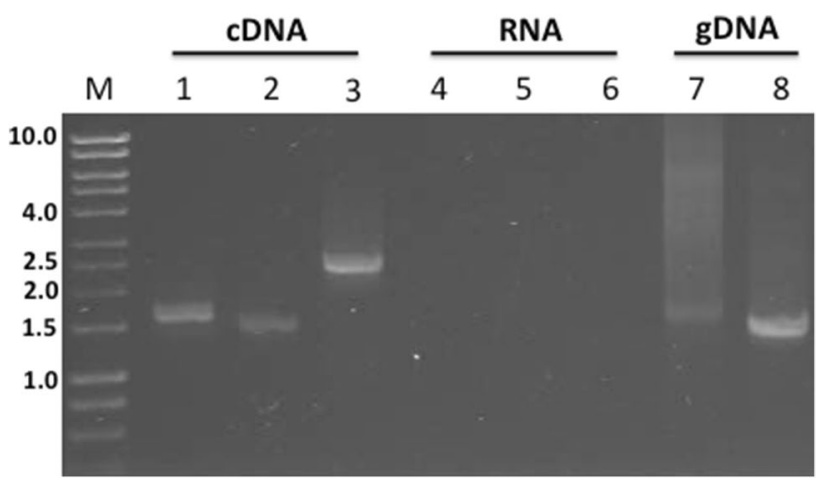

Fig. 3 Analysis of the presence and gene expression of trans-sialidase gene in the bloodstream stages of $T . b$. brucei. Conventional PCR products were subjected to electrophoresis on agarose gel $(1 \% \mathrm{w} / \mathrm{v})$ in TAE buffer, ethidium bromide, and visualized using ultraviolet light. Lanes 1-3: PCR products obtained from cDNA as template; lanes 4-6: PCR product obtained from total RNA as template negative control; lanes 7-8: PCR product obtained from genomic DNA. Both templates were isolated from bloodstream stages of $T . b$. brucei. $M$ molecular marker-bp (Bioline-UK), gDNA genomic DNA

(RT-PCR and conventional PCR), recombinant TS protein was produced in Escherichia coli bacteria. Next, in order to use the recombinant TS protein as a possible serological 
marker for AT, T. b. brucei TS gene was cloned into a gene expression plasmid for bacterial gene expression system (results not shown).

The constructed plasmid DNA, denominated nTSpET28a, was used to transform BL21 (DE3) E. coli bacteria and subsequently to express and produce nTS recombinant protein using IPTG induction. Using SDS-PAGE technique, bands corresponding to nTS recombinant protein (about $52 \mathrm{kDa}$ ) were obtained (Fig. 4). The nTS recombinant protein, represented by the arrow in the Fig. 4, was purified by removing the band from the SDS-PAGE gel, according to the protocol presented in the session materials and methods (results not shown). Afterwards, purified nTS recombinant protein was subsequently immunoidentified by ELISA and immunoblotting (result not shown).

In order to determine the presence of anti-TS antibodies in the mice infected with $T . b$. brucei, blood samples were collected at $0,5,14,21,25$, and 45 days post-infection and sera were obtained. The course of infection was determined by parasitemia in the blood, visualized by optical microscopy (results not shown) and by the presence of total IgG antibodies anti-T. b. brucei, determined by ELISA, as shown in Fig. 5. Sera from infected Balb-C mice show an increase in titter of IgG antibodies that specifically recognize a crude extract of bloodstream forms of T. b. brucei. We established the experimental model of infection with $T . b$. brucei through the presence of parasites in the blood and the presence of anti-T. $b$. brucei antibodies by ELISA.

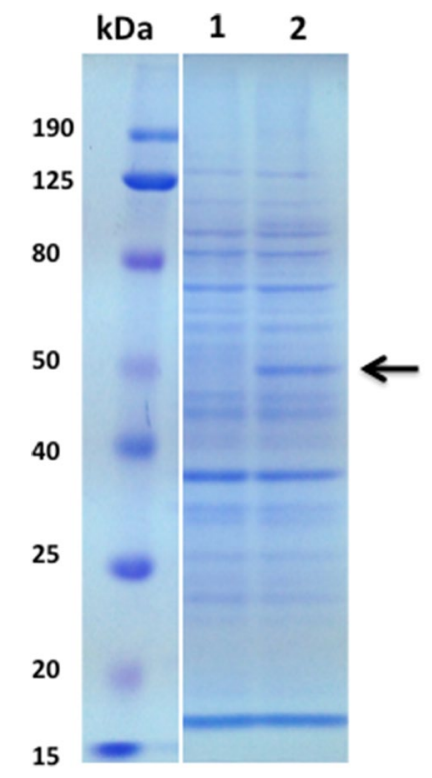

Fig. 4 Gene expression of recombinant nTS protein produced in $E$. coli BL21 (DE3) and analysed by SDS-PAGE. Lanes 1 and 2 represent the electrophoretic protein profile of E. coli BL21 (DE3) without and with IPTG induction, respectively

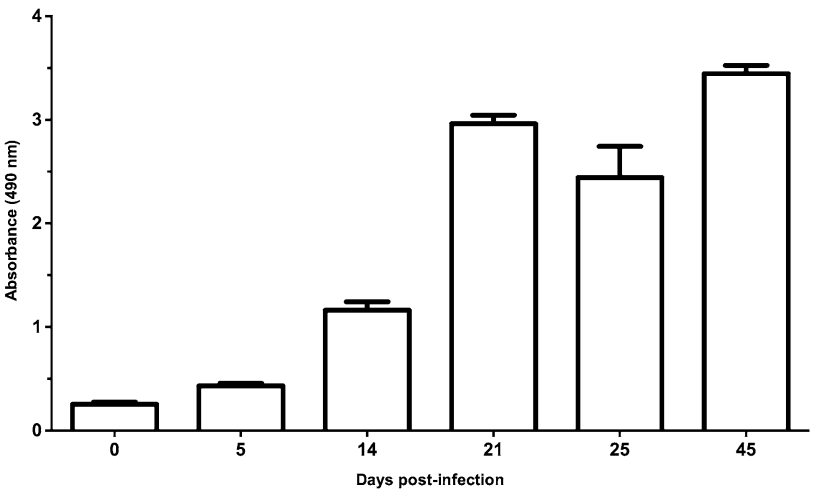

Fig. 5 Determination of total IgG anti-T. b. brucei antibodies in sera from infected mice with $T$. $b$. brucei parasites. Bars represent $\mathrm{IgG}$ obtained by ELISA from diluted sera (1:200), measured by optical density (absorbance) at different time points post infection. Each bar represents the mean and standard deviation of the absorbance values obtained in duplicate from each group of infected animals $(n=3)$

In order to determine the presence of anti-TS antibodies in mice experimentally infected with $T$. $b$. brucei, nTS recombinant protein was used for the determination of anti-nTS antibodies. ELISA was performed to measure the titters of total IgG anti-nTS, as well as the IgG1, IgG2a and IgG3 subtypes (Fig. 6). The titters of IgG anti-nTS antibodies increased with the course of infection caused by $T$. $b$. brucei, as the same was observed for IgG anti-T. b. brucei (Fig. 5). In Fig. 6 it is also possible to observe the profile of anti-nTS subtypes antibodies (IgG1, IgG2a and $\mathrm{IgG} 3)$.

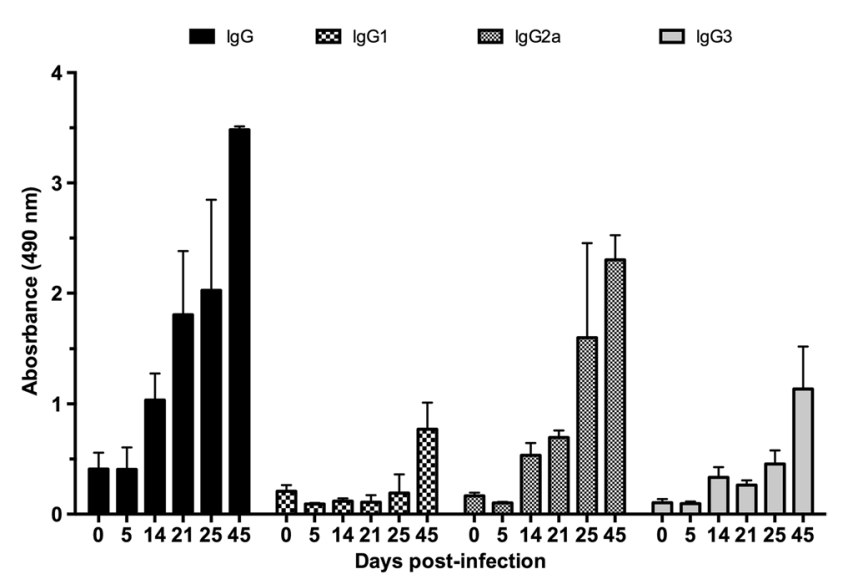

Fig. 6 Determination of total and subtypes IgGs antibodies against trans-sialidase recombinant protein in sera from infected mice with $T$. b. brucei parasites. The horizontal axis represents days post infection versus optical density (absorbance) obtained by ELISA performed with 1:200 sera dilution. Each bar represents the mean and standard deviation of the absorbance values obtained in duplicate from each group of infected animals $(n=3)$ 


\section{Discussion}

African trypanosomiasis, caused by the flagellated protozoan T. brucei, is a neglected tropical disease with great impact on human and animal health. This infectious disease can be found in 36 countries of sub-Saharan African, where there are tsetse flies that transmit the parasite. Although recently the number of new cases of human disease has significantly been reduced [21], AT still affects millions of people and animals, contributing for economic disruption specially in rural communities, representing a important factor responsible for the delay in the development of the affected regions [22, 23].

Control programmes implemented in the context of AT are generally composed by diagnosis and treatment of new clinical cases. However, drugs used in the treatment against $T$. brucei are ineffective and present too much toxicity; diagnosis and treatment of the disease is complex and requires specifically skilled staff and laboratory diagnostic methods have low specificity and sensitivity. Considering the difficulties that both diagnostic and treatment present, it is necessary to identify potential new targets that can be used for the development of new drugs and new diagnostic markers for the control of AT.

The main purpose of this work is to use trans-sialidase protein (TS) as a possible serological marker in infections caused by $T$. brucei, and to demonstrate the presence of the TS in the bloodstream stages of T. b. brucei. It is important to highlight that, until now, the TS enzyme has only been described in the procyclic forms of T. brucei. Thus, we could enhance the use of TS as a possible serological marker in infections caused by $T$. brucei.

The expression and presence of TS protein in bloodstream forms of $T$. brucei is controversial and has only been described in the procyclic form, a situation in which the parasites replicate in the invertebrate host [4-6]. Procyclins proteins cover the procyclic forms of $T$. brucei. Procyclins have an uncommon GPI anchor that, unlike the GPI anchor of VSGs, is composed by poly- $N$-acetyllactosamine repeats capped by sialic acid residues $[4,5]$. Nakatani et al. [24] suggest that TS protein plays a role in the sialic acid modification of the procyclic stage $T$. brucei cell surface.

The sialic acid contributes for the biological properties of cell surface. In $T$. cruzi parasites, the etiological agent of Chagas disease, the sialic acid has been implicated in cell-cell interactions and T-cell activation, a key for their survival in the blood [25]. It has been suggested that sialylated molecules are involved in the invasion of mammalian host cells and in the protection from complement lysis [5]. Furthermore, Trypanosomes, that do not acquire sialic acids due to a defect in the production of the sialylated GPI anchors, do not survive in the intestine of the vector. Thus, the sialic acid present on cell surface appears to protect parasites from the environment in the midgut of the tsetse fly [26].

In this work we demonstrated that bloodstream forms of T. b. brucei, purified from blood of infected mice, express the TS protein. This finding was obtained using cDNA produced from RNA extracted from bloodstream forms of $T$. $b$. brucei purified from infected mice. Interestingly, the presence of the TS protein in the bloodstream forms of $T$. b. brucei was demonstrated indirectly through the production of anti-TS IgG antibodies in $T$. $b$. brucei infected mice, and by immunoblotting (results not shown). However, the biological function and molecular characterization of the TS protein from bloodstream stages of T. $b$. bruce $i$ are not widely known and investigated.

In a previous work, we hypothesized that TS could be expressed in the bloodstream stages of $T$. b. brucei. Our work has showed that mice vaccinated with plasmid DNA encoding the 5'-terminal region of the trans-sialidase (nTS) gene of $T . b$. bruce $i$ were able to produce IgG antibodies that bind to the bloodstream forms of $T$. $b$. brucei, and consequently recognize the recombinant nTS protein [20]. These findings allowed us to suggest that TS protein (or unlikely a TSA-like cross-reactive protein) was also expressed in the bloodstream forms of T. b. brucei, in similarity to what happens with $T$. cruzi $[8,9]$.

Our results suggest for the first time that TS protein is expressed in the circulating bloodstream forms of $T$. $b$. brucei, thus constituting a potential antigenic target, up until now not considered. Nevertheless, a more detailed study is required regarding the levels of mRNA expression in the bloodstream forms of T. b. brucei. In this study, we report a preliminary reverse transcriptase-PCR (RT-PCR) study to analyse the presence of TS mRNA. However, a quantitative PCR will be required to determine TS mRNA levels in these bloodstreams forms of T. b. brucei.

In this work, we believe that TS bloodstream protein may be a potential antigen target due to its ability to generate antibodies during the course of infection caused by $T$. b. brucei, and may be an important antigen, responsible for the induction of the immune response during the parasitehost interaction. It is also important to highlight the type of anti-TS antibodies generated during AT, preferably Th1type antibodies (IgG2a and IgG3). This pattern of immune response is expected since $T . b$. brucei stimulates a Th1 immune response with increased titres of $\operatorname{IgG} 2 \mathrm{a}$. With this finding a whole new need is emerging: to investigate and understand the biological role of TS protein in the bloodstream stages of T. b. brucei. 


\section{Materials and Methods}

\subsection{Parasites and Mice}

Strain T. b. brucei GVR 35/1.5 was used for experimental infection of AT in murine model [27]. Animals used in this work were female Balb/C mice between 5 and 8 weeks old, obtained from Institute of Hygiene and Tropical Medicine, Lisbon-Portugal.

\subsection{Experimental Model of T. b. brucei}

For experimental AT infection, mice were inoculated intraperitoneally with approximately 500 parasites, prepared by dilution of the infected blood with PBS-glucose $20 \mathrm{mM} \mathrm{pH}$ 7.4. The murine model of infection with $T . b$. brucei is extensively used in our laboratory [19, 28, 29]. Serum samples from $T . b$. brucei-infected mice were obtained at $0,5,14$, 21,25 and 45 days post-infection. All sera were stored at $-20{ }^{\circ} \mathrm{C}$ until use. Next, serum samples were used for the detection of anti-T. b. brucei and anti-TS protein antibodies by ELISA.

\subsection{Gene Cloning and Protein Expression of Trans-sialidase}

Plasmid DNA (nTSpVAX1), previously constructed by Silva et al. [19], was used as template for nTS gene amplification by PCR. The reaction was performed with $10 \mathrm{ng}$ of plasmid nTSpVAX1, 100 pmol of primers, $0.5 \mathrm{mM}$ of mix dNTPs (Bioline-Germany), $3 \mathrm{mM} \mathrm{MgCl} 2,5 \mathrm{U}^{\text {Biotaq }}{ }^{\mathrm{TM}}$ DNA polymerase (Bioline-Germany), and NH4 buffer (BiolineGermany). Primers sense (5'ATTATAGCTAGCATGGAG GAACTCCACCAAC'3) and anti-sense (5'TAATCCCTT AAGTCAGTGCAGACAATAA'3) were designed to amplify about $1416 \mathrm{bp}$ from the trans-sialidase gene [5], here referred as nTS gene. The PCR amplicon was enzymatically digested by NheI and EcoRI endonucleases (Fermentas-USA) and cloned in the pET28a plasmid (Novagen - USA). The constructed plasmid (nTSpET28a) was submitted to restriction analysis and sequencing (data not shown).

For gene expression of the recombinant nTSA protein, competent cells $E$. coli BL21 (DE3) were transformed with nTSpET28a by heat shock. The cells were selected with kanamycin antibiotic (Bioline-UK) and expanded with agitation at $350 \mathrm{rpm}$ in $\mathrm{LB}$ medium at $37^{\circ} \mathrm{C}$ up to approximately $0.8-1$ optical density absorbance $(600 \mathrm{~nm})$. The nTS gene expression was induced by adding $0.5 \mathrm{mM}$ of isopropylbD-1-thiogalactopynaroside (IPTG) for $18 \mathrm{~h}$ at $37{ }^{\circ} \mathrm{C}$ and 350-rpm agitation. Cells were then centrifuged, pelleted and suspended in buffer saline (PBS pH 7.4). SDS-sample buffer (v/v) was added, and this mixture was reduced for $5 \mathrm{~min}$ in a boiling water bath. Samples were then submitted to sodium SDS-PAGE. The partial purification of nTS recombinant protein was preceded according to established protocols [30]. The partial purified proteins were quantified and visualised by SDS-PAGE stained with Coomassie blue R-250 to confirm the presence of the partial purified protein. The partial purified nTS protein was used as the antigen source for the serological assay (ELISA) and immunoblotting.

\subsection{Identification of the Trans-sialidase Expression in the Bloodstream T. b. brucei}

Blood samples from T. $b$. brucei-infected mice were collected and used for the RNA extraction, according to RNA isolation Kit, (Bioline, UK). Concentration of total RNA was determined by NanoDrop 1000 (Thermo Scientific). The cDNA synthesis was performed according to the cDNA Synthesis Kit (Bioline, UK). Complementary DNA was used as template for nTS gene amplification by polymerase chain reaction (PCR) assay. The reaction was performed with $100 \mathrm{ng}$ of cDNA, $100 \mathrm{pmol}$ of primers, $0.5 \mathrm{mM}$ of mix dNTPs (Bioline-Germany), $3.0 \mathrm{mM} \mathrm{MgCl} 2,5.0 \mathrm{U}$ Biotaq $^{\mathrm{TM}}$ DNA polymerase (Bioline-Germany), and NH4 buffer (Bioline-Germany). The same specific primers, previously described above, were used to amplify the first 1,416 bp from the TS gene (designated nTSA gene). Reaction mixtures were incubated in a thermocycler (MJ-Mini Bio-RadSingapure) at $94{ }^{\circ} \mathrm{C}$ for $2 \mathrm{~min}$, followed by 40 cycles of $30 \mathrm{~s}$ at $94{ }^{\circ} \mathrm{C}, 1 \mathrm{~min}$ and $30 \mathrm{~s}$ at $60{ }^{\circ} \mathrm{C}$, and $2 \mathrm{~min}$ at $72{ }^{\circ} \mathrm{C}$. After the final cycle, reaction mixtures were cooled down at $4{ }^{\circ} \mathrm{C}$. The amplified product was examined by performing a $1 \%$ agarose gel electrophoresis ran at $100 \mathrm{~V}$ in TAE buffer (40 mM Tris base, $20 \mathrm{mM}$ acetic acid, $\mathrm{pH}$ 8.0, $1 \mathrm{mM}$ EDTA), stained with ethidium bromide.

\subsection{Determination of Anti-T. b. brucei and Anti-trans-sialidase Antibodies by Enzyme-Linked Immunosorbent Assay}

Total protein of bloodstream forms of $T . b$. bruce $i$ and partially purified nTS recombinant protein were used to adsorb 96-well micro-plates to perform indirect enzyme-linked immunosorbent assays (ELISA), using sera from T. b. brucei-infected mice. Total protein of bloodstream forms of $T$. $b$. brucei was obtained from heparinized total blood collected from infected mice. The parasites were purified using a gravity-operated DEAE-cellulose chromatography column described by Lanham and Godfrey [31].

Total serum immunoglobulin $\mathrm{G}$ (IgG) specific for $T$. $b$. $b r u$ $c e i$ and for nTSA recombinant protein was measured. Additionally, IgG1, IgG2a and IgG 3 antibodies were also determined. All sera from infected mice were assayed in duplicate 
and specific antibodies measured at $490 \mathrm{~nm}$. Previously, 96-well micro-plates (BD Falcon-USA) were coated with $100 \mathrm{ng} /$ well of partial purified nTSA in carbonate buffer $\mathrm{pH} 8.5$ (overnight at $4{ }^{\circ} \mathrm{C}$ ). The micro-plates were washed three times with PBS-0.05\% Tween20 (Promega-USA) and blocked for one hour at room temperature with $1 \%(\mathrm{w} / \mathrm{v})$ bovine serum albumin solution in PBS. After three washes, serial dilution of infected mice sera were added for $1 \mathrm{~h}$ at room temperature with horseradish peroxidase (HRP)-conjugated rat anti-mouse IgG (diluted 1:4000 v/v-Sigma Chemical Co-USA), rat antimouse IgG1 (500 ng/mL-AbDserotec-UK), rat anti-mouse $\operatorname{IgG} 2 \mathrm{a}(250 \mathrm{ng} / \mathrm{mL}$-AbDserotec-UK) and rat anti-mouse IgG3 (diluted 1:4000 v/v-AbDserotec-UK). The HRPconjugated antibodies were incubated for $30 \mathrm{~min}$ with a substrate solution $(10 \mathrm{~mL}$ of citrate buffer $\mathrm{pH} 5.0$ with $10 \mathrm{mg}$ of OPD and $10 \mu \mathrm{L}$ of hydrogen peroxide $30 \%$-Sigma-USA). Then, sulphuric acid $4 \mathrm{~N}$ was used as stop solution and absorbance was determined $490 \mathrm{~nm}$ by spectrometry. This assay was developed according to the protocol developed by [20].

\section{Conclusions}

We concluded that during the course of infection caused by T. b. brucei, mice show increased titters of antibodies specific to TS protein, represented herein as nTS recombinant protein. These data open the opportunity to evaluate the biological function of TS protein during the parasite-host interaction process - a serological marker and a potential target for vaccine development in the context of AT.

Acknowledgements Thanks to Global Health and Tropical Medicine (GHTM-UID/multi/04413/2013) by support and the Programa Ciências Sem Fronteiras, Capes-Brazil (Grant No. 019/2013). We are also grateful to Paulo Fanado for editing this manuscript.

Author Contributions Conceived and designed the experiments: AFT and MSS; Performed the experiments: AFT, JGP, and SPA; Analysed the data: AFT, JGP, SPA, and MSS; Wrote the paper: AFT and MSS. All authors read and approved the final version of the manuscript.

\section{Compliance with Ethical Standards}

Conflict of interest The authors declare no conflict of interest.

Ethics Approval Murine model was performed in accordance with existing legislation in Portugal and in accordance of the Ethical Committee at Institute of Hygiene and Tropical Medicine, Lisbon-Portugal.

\section{References}

1. Matthews KR, Ellis JR, Paterou A (2004) Molecular regulation of the life cycle of African trypanosomes. Trends Parasitol 20(1):40-47
2. Cardoso de Almeida ML, Turner MJ (1983) The membrane form of variant surface glycoproteins of Trypanosoma brucei. Nature 302(5906):349-352

3. Ponte-Sucre A (2016) An overview of Trypanosoma brucei infections: an intense host-parasite interaction. Front Microbiol 7(2126):1-12

4. Montagna GN, Donelson JE, Frasch AC (2006) Procyclic Trypanosoma brucei expresses separate sialidase and transsialidase enzymes on its surface membrane. J Biol Chem 281(45):33949-33958

5. Montagna G, Cremona ML, Paris G, Amaya MF, Buschiazzo A, Alzari PM, Frasch AC (2002) The trans-sialidase from the African trypanosome Trypanosoma brucei. Eur J Biochem 269(12):2941-2950

6. Engstler M, Reuter G, Schauer R (1993) The developmentally regulated trans-sialidase from Trypanosoma brucei sialylates the procyclic acidic repetitive protein. Mol Biochem Parasitol 61(1):1-13

7. Pontes de Carvalho, LC, Tomlinson S, Vandekerckhove F, Bienen EJ, Clarkson AB, Jiang MS, Hart GW, Nussenzweig V (1993) Characterization of a novel trans-sialidase of Trypanosoma brucei procyclic trypomastigotes and identification of procyclin as the main sialic acid acceptor. J Exp Med 177(2):465-474

8. Schenkman S, Jiang MS, Hart GW, Nussenzweig V (1991) A novel cell surface trans-sialidase of Trypanosoma cruzi generates a stage-specific epitope required for invasion of mammalian cells. Cell 65(7):1117-1125

9. Schankman S, Eichinger D (1993) Trypanosoma cruzi transsialidase and cell invasion. Parasitol Today 9(6):218-222

10. Egima CM, Briones MR, Freitas Júnior LH, Schenkman RP, Uemura H, Schenkman S (1996) Organizaton of trans-sialidase genes in Trypanosoma cruzi. Mol Biochem Parasitol 77(2):115-125

11. Rubin-de-Celis SS, Uemura H, Yoshida N, Schenkman S (2006) Expression of trypomastigote trans-sialidase in metacyclic forms of Trypanosoma cruzi increases parasite escape from its parasitophorous vacuole. Cell Microbiol 8(12):1888-1898

12. Perreira-Chioccola VL, Fragata-Filho AA, Levy AM, Rodrigues MM, Schenkman S (2003) Enzyme-linked immunoassay using recombinant trans-sialidase of Trypanosoma cruzi can be employed for monitoring of patients with Chagas' disease after drug treatment. Clin Diagn Lab Immunol 10(5):826-830

13. Ribeirão M, Pereira-Chioccola VL, Rénia L, Filho AF, Schenkman S, Rodrigues MM (2000) Chagasic patients develop a type 1 immune response to Trypanosoma cruzi trans-sialidase. Parasite Immunol 22(1):49-53

14. Costa F, Franchin G, Pereira-Chioccola VL, Ribeirão M, Schenkman S, Rodrigues MM (1998) Immunization with a plasmid DNA containing the gene of trans-sialidase reduces Trypanosoma cruzi infection in mice. Vaccine 16(8):768-774

15. Schauer R, Kamerling JP (2011) The chemistry and biology of trypanosomal trans-sialidases: virulence factor in Chagas disease and sleeping sickness. Chembiochem 12(15):2246-2264

16. Engstler M, Schauer R, Brun R (1995) Distribution of developmentally regulated trans-sialidases in the Kinetoplastida and characterization of a shed trans-sialidase activity from procyclic Trypanosoma congolense. Acta Trop 59(2):117-129

17. Tiralongo E, Martensen I, Grötzinger J, Tiralongo J, Schauer R (2003) Trans-sialidase-like sequences from Trypanosoma congolense conserve most of the critical active site residues found in other trans-sialidases. Biol Chem 384(8):1203-1213

18. Tiralongo E, Schrader S, Lange H, Lemke H, Tiralongo J, Schauer R (2003) Two trans-sialidase forms with different sialic acid transfer and sialidase activities from Trypanosoma congolense. J Biol Chem 278(26):23301-23310 
19. Ammar Z, Plazolles N, Baltz T, Coustou V (2013) Identification of trans-sialidases as a common mediator of endothelial cell activation by African trypanosomes. PloS Pathog 9(10):e1003710. https://doi.org/10.1371/journal.ppat.1003710

20. Silva MS, Prazeres DM, Lança A, Atouguia J, Monteiro GA (2009) Trans-sialidase from Trypanosoma brucei as a potential target for DNA vaccine development against African trypanosomiasis. Parasitol Res 105(5):1223-1229

21. World Health Organization (2007) Report of a WHO informal consultation on sustainable control of human African trypanosomiasis. Geneva, Switzerland

22. Giordani F, Morrison LJ, Rowan TG, Koning DE, Barrett HP, M. P (2016) The animal trypanosomiasis and their chemotherapy: a review. Parasitology 143(14):1862-1889

23. Lejon V, Jacobs J, Simarro PP (2013) Elimination of sleeping sickness hindered by difficult diagnosis. Bull World Health Organ 91(10):718

24. Nakatani F, Morita YS, Ashida H, Nagamune K, Maeda Y, Kinoshita T (2011) Identification of a second catalytically active trans-sialidase in Trypanosoma brucei. Biochem Biophys Res Commun 415(2):421-425

25. Tribulatti MV, Mucci J, Van Rooijen N, Leguizamón MS, Campetella O (2005) The trans-sialidase from Trypanosoma cruzi induces thrombocytopenia during acute Chagas'disease by reducing the platelet sialic acid contents. Infect Immun 74(1):201-207

26. Nagamune $\mathrm{K}$, Acosta-Serrano A, Uemura H, Brun R, Kunz-Renggli C, Maeda Y, Ferguson MA, Kinoshita T (2004) Surface sialic acids taken from the host allow trypanosome survival in tsetse fly vectors. J Exp Med 199(10):1445-1450

27. Jennings FW (1993) Combination chemotherapy of CNS trypanosomiasis. Acta Trop 54:205-213

28. Lança AS, de Sousa KP, Atouguia J, Prazeres DM, Monteiro GA, Silva MS (2011) Trypanosoma brucei: immunisation with plasmid DNA encoding invariant surface glycoprotein gene is able to induce partial protection in experimental African trypanosomiasis. Exp Parasitol 127(1):18-24

29. de Sousa KP, Atouguia J, Silva MS (2010) Partial biochemical characterization of a metalloproteinase from the bloodstream forms of Trypanosoma brucei brucei parasites. Protein $\mathrm{J}$ 29(4):283-289

30. Retamal CA, Thiebaut P, Alves EW (1999) Protein purification from polyacrylamide gels by sonication extraction. Anal Biochem 268:15-20

31. Lanham SM, Godfrey DG (1970) Isolation of salivarian trypanosomes from man and other mammals using DEAE-cellulose. Exp Parasitol 28(3):521-534

Publisher's Note Springer Nature remains neutral with regard to jurisdictional claims in published maps and institutional affiliations. 